Supporting Information for

\title{
Integrated Microfluidic System for Size-Based Selection and Trapping of Giant Vesicles
}

\author{
Yuki Kazayama", Tetsuhiko Teshima , Toshihisa Osaki\$, Shoji Takeuchi**, \\ Taro Toyota ${ }^{\dagger} * *$ \\ † Graduate School of Arts and Sciences, The University of Tokyo, 3-8-1 Komaba, \\ Meguro-ku, Tokyo 153-8902, Japan. E-mail: cttoyota@mail.ecc.u-tokyo.ac.jp \\ $\$$ Institute of Industrial Science (IIS), The University of Tokyo, 4-6-1 Komaba, \\ Meguro-ku, Tokyo 153-8505, Japan. E-mail: takeuchi@iis.u-tokyo.ac.jp. \\ $\S$ Kanagawa Academy of Science and Technology, Kanagawa, Japan. \\ *** Co-corresponding authors.
}

\section{Table of Contents}

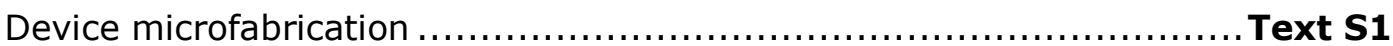

Liposome formation and used lipids..................................... Text $\mathbf{S 2}$

Image acquisition and data treatment ................................ Text S3

A schematic illustration of the microfluidic system........................ Figure S1

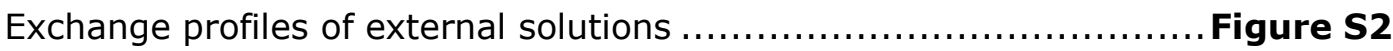

Size distributions of trapped liposomes analyzed using ImageJ.......... Figure S3

Theoretical prediction of trapping state change ........................ Figure $\mathbf{S 4}$

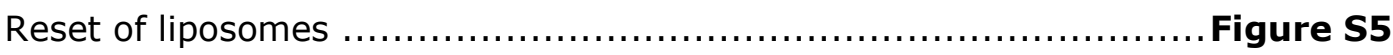




\section{Text S1. Device microfabrication}

The microfluidic device was fabricated as follows. A chrome plating photomask was fabricated using a maskless lithography system (Nano System Solutions, D-light DLS-50). An SU8 (MicroChem Co., MA, USA) master mold on a silicon wafer was then obtained using an ultraviolet (UV) lithography process with the photomask. The thickness of the mold was designed according to the target diameters listed in Table 1. Microfluidic devices

of polydimethylsiloxane (PDMS) were obtained from the master mold. The PDMS part of the device was bonded to a glass slide (Matsunami Glass Ind., $0.25-0.35 \mathrm{~mm}$ in thickness) using $\mathrm{O}_{2}$ plasma treatment (SAMCO, FA-1). 
Text S2. Liposome formation and used lipids.

\section{Materials}

1-Palmitoyl-2-oleoyl-sn-glycero-3-phosphocholine (POPC) and 1-palmitoyl-2-oleoyl-snglycero-3-[phospho-rac-(1-glycerol)] (POPG) were purchased from NOF Corporation (Tokyo, Japan), and cholesterol was provided by Avanti Polar Lipids (Alabaster, AL, USA). Green fluorescent lipid, 2-(4,4-difluoro-5,7-dimethyl-4-bora-3a,4a-diaza-s-indacene-3dodecanoyl)-1-hexadecanoyl-sn-glycero-3-phosphocholine (BODIPY-HPC), was purchased from Life Technologies (Gaithersburg, MD, USA). Fructose, uranine, chloroform, and methanol were purchased from Wako Pure Chemical Industry Ltd. (Osaka, Japan). Milli-Q water (Millipore reagent water system, Bedford, MA, USA) was used throughout the experiments.

\section{Preparation of liposomes}

The liposome suspension was prepared as follows. POPC, POPG, cholesterol (Chol), BODIPY-HPC, and fructose (Fru) were dissolved in a chloroform/methanol solution (9:1, $\mathrm{v} / \mathrm{v})$. A clean disposable glass vial containing $500 \mu \mathrm{L}$ of the mixture was rotated by a rotary evaporator (EYELA, N1110V), and a lipid film was prepared by evaporating the organic solvents. The remaining solvents were completely removed under reduced pressure over a period of more than $2 \mathrm{~h}$. Then, $2 \mathrm{~mL}$ of Milli-Q water was poured into the vial, and the dry lipid film was hydrated in a thermostatic incubator (WAKENYAKU, MODEL 2290) at $40^{\circ} \mathrm{C}$ for more than $1 \mathrm{~h}$ with gentle agitation. The final concentration of POPC/POPG/Chol/BODIPY-HPC/Fru after swelling was 1:0.11:0.11:0.005:1 in milliMolarity units. Finally, the liposome suspension was filtered using a nylon mesh filter (Millipore) to inhibit aggregation in a microfluidic channel. The pore sizes of the filters were $20 \mu \mathrm{m}$ for target diameters of 12 and $16 \mu \mathrm{m}$, and $30 \mu \mathrm{m}$ for the target diameter of $20 \mu \mathrm{m}$. 


\section{Text S3. Image acquisition and data treatment}

\section{Image acquisition}

Microscopic images of liposomes at the trapping region were collected using an inverted microscope (Olympus, IX71) with a cooled charge-coupled device (CCD) camera (Olympus, DP72) or a confocal scanning unit (Yokogawa Electric, CSU22) equipped with a scientific complementary metal-oxide semiconductor (sCMOS) camera (Andor, Zyla).

\section{Data treatment}

To analyze the size distribution of the trapped liposomes and quantify the morphological changes in the liposome under sequential osmotic stresses, we used a custom program based on Open $\mathrm{CV}$, which is an open source computer vision library. ${ }^{1}$ The mean diameter was calculated from the area enclosed by the contour of a binarized image of the trapped liposomes. In the osmotic stress experiments, the major axis was measured using the horizontal distance between liposome membranes. In this study, we defined the nominal lamellarity as the ratio of the area of the internal membrane structures and the area inside the respectively evaluated liposome.

(1) An open source computer vision and machine learning software library. Available: http://opencv.org/ 


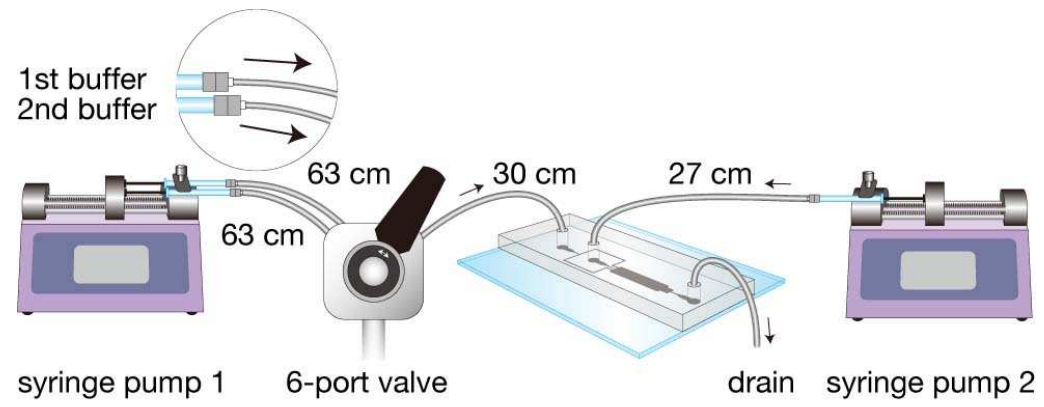

Figure S1. A schematic illustration of the microfluidic system.

The experimental setup of the microfluidic device and pumps is shown schematically in Figure S1. 

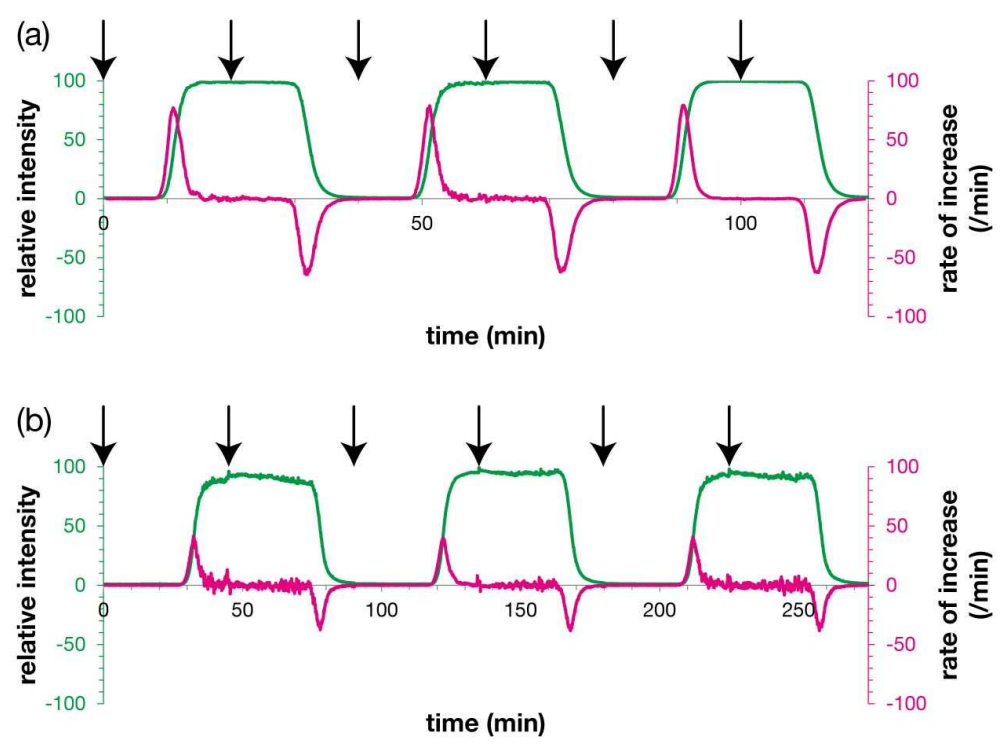

Figure S2. Exchange profiles of external solutions (fluorophore: uranine). Flow rates were set to $330 \mu \mathrm{L} / \mathrm{h}$ (a) and $105 \mu \mathrm{L} / \mathrm{h}$ (b). External solutions were switched at the times depicted by solid arrows.

The exchange rate for the external solutions was measured from the fluorescence intensities using a uranine solution. The changes in the fluorescence intensity over time and the rate of increase are represented in Figure S2. A rapid exchange of external solutions was achieved under a high flow rate. However, a low flow rate was desirable for decreasing the flow stresses of the trapped liposomes and keeping them stable. Therefore, we combined the switching of the external solutions with the control of the volumetric flow rate to ensure both a rapid and low-stress exchange of the external solutions. As a result, it took only about $19 \mathrm{~min}$ (the fluorescence intensity reached $80 \%$ of its maximum) to exchange the external solutions $(330 \mu \mathrm{L} / \mathrm{h})$, and the gradual contraction of the trapped liposomes was successfully observed under the condition of a low flow rate $(105 \mu \mathrm{L} / \mathrm{h})$. 

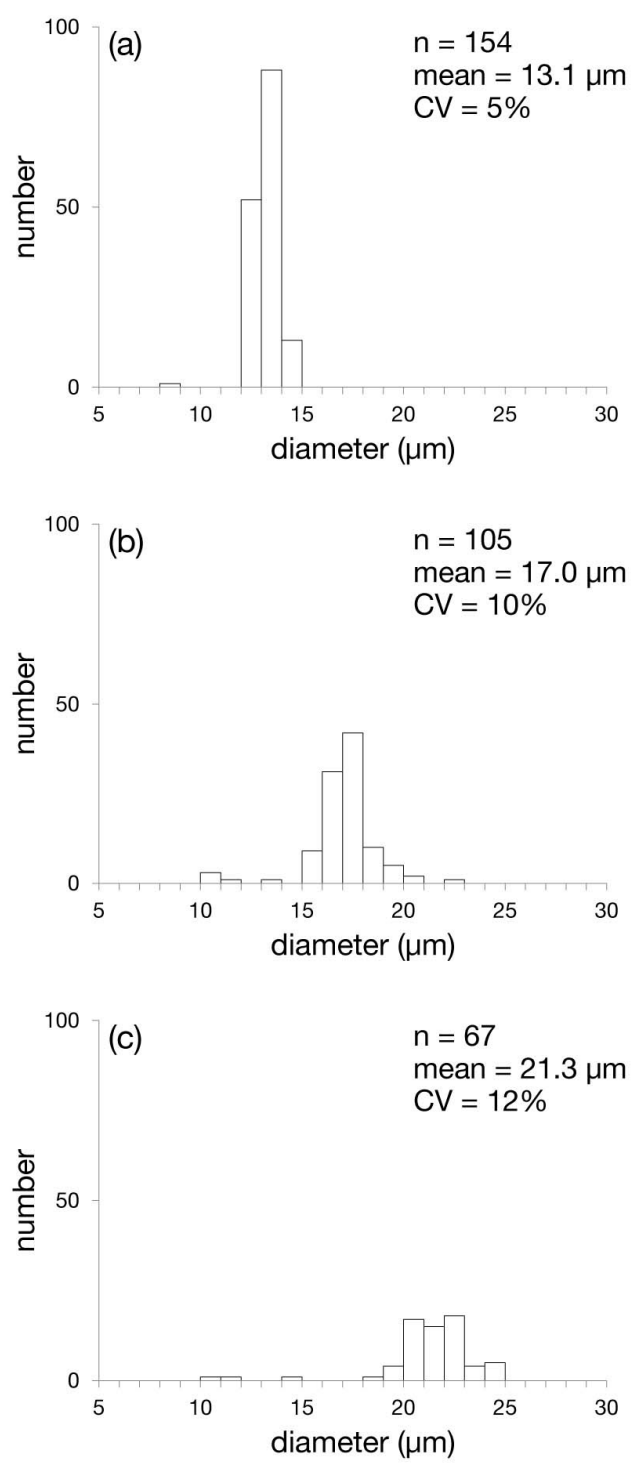

Figure S3. Size distributions of trapped liposomes analyzed using ImageJ. The target diameters were $12 \mu \mathrm{m}$ (a), $16 \mu \mathrm{m}$ (b), and $20 \mu \mathrm{m}$ (c).

The size distributions of the trapped liposomes analyzed using the ImageJ software are shown in Figure S3. The mean diameters and CVs of the trapped liposomes were $13.1 \mu \mathrm{m}$, $5 \%(\mathrm{n}=154), 17.0 \mu \mathrm{m}, 10 \%(\mathrm{n}=105)$, and $21.3 \mu \mathrm{m}, 12 \%(\mathrm{n}=67)$ for target diameters of 12 , 16 , and $20 \mu \mathrm{m}$, respectively. In the case of the Open CV operations, binarization tended to generate a slightly large area that completely enclosed the trapped liposomes. In contrast, in the case of the ImageJ software operations, ellipses were drawn by hand to preferably fit along the center of the liposome membrane. Thus, the mean diameters of the trapped liposomes analyzed by the ImageJ software were smaller. However, we found that they were still larger than the theoretical predictions. 


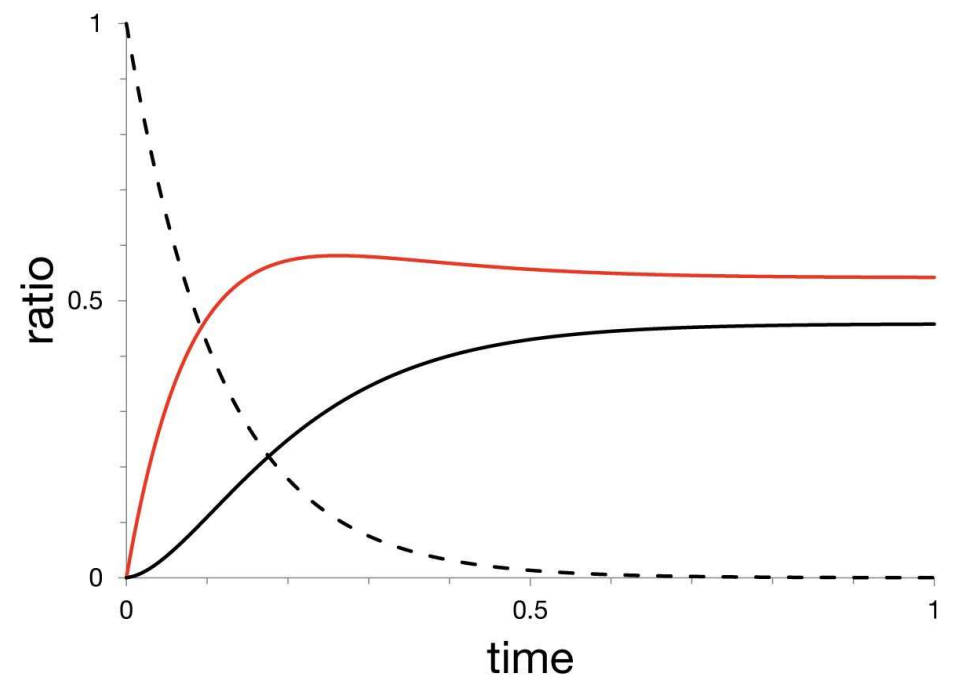

Figure S4. Theoretical prediction of trapping state change. The ratios of the successful traps (solid red line), blank traps (dashed black line), and error traps (solid black line) were calculated for the parameter set $\left(k_{1}, k_{1}{ }^{\prime}, k_{2}, k_{2}{ }^{\prime}\right)$ $=(90,0,44,52)$.

The transition rates were determined as follows.

$$
\begin{aligned}
\frac{d\left[S_{0}(t)\right]}{d t}= & -k_{1}\left[S_{0}(t)\right]+k_{1}^{r}\left[S_{1}(t)\right] \\
\frac{d\left[S_{1}(t)\right]}{d t}= & k_{1}\left[S_{0}(t)\right]-k_{1}^{r}\left[S_{1}(t)\right]-k_{2}\left[S_{1}(t)\right]+k_{2}^{r}\left[S_{2}(t)\right], \\
& \frac{d\left[S_{2}(t)\right]}{d t}=k_{2}\left[S_{1}(t)\right]-k_{2}^{r}\left[S_{2}(t)\right] .
\end{aligned}
$$

The number of trapped liposomes in each trapping state was counted, and each proportional constant was estimated from nine pairs of neighboring fluorescence images. Then, the ratio of each trapping state was numerically calculated using a backward-Euler method with the following conditions.

$$
\begin{gathered}
{\left[S_{0}(0)\right]=1, \quad\left[S_{1}(0)\right]=\left[S_{2}(0)\right]=0 .} \\
{\left[S_{0}(t)\right]+\left[S_{1}(t)\right]+\left[S_{2}(t)\right]=1}
\end{gathered}
$$

Figure S4 shows a typical prediction of the trapping state change normalized over time. The result indicates that the ratio of successful traps changes along a curve with a local maximum and reaches a stable value. This trend is consistent with our experimental results. In the case of the target diameter of $20 \mu \mathrm{m}$, the ratio of successful traps reached its maximum value during observation. In contrast, in the cases of target diameters of 12 and $16 \mu \mathrm{m}$, we observed only the plateau regions. 

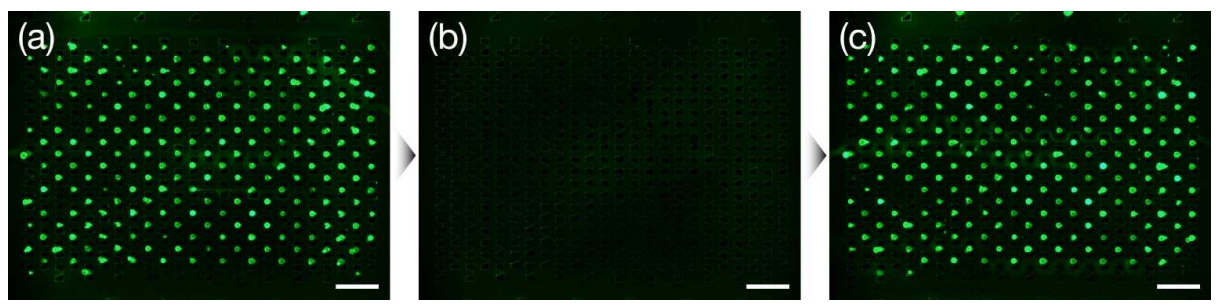

Figure S5. Reset of liposomes. Monodisperse liposomes were set at the first loading step (a) and completely flushed out under a high flow rate (b). Liposomes were successfully reset at the second loading step (c). The scale bars represent $100 \mu \mathrm{m}$.

A deformability-based microfluidic cell pairing technique was previously reported. ${ }^{2}$ Cells could be deformed at a flow rate 50 times higher than the trapping rate. In contrast, liposomes were completely flushed out at a lower flow rate (12 times higher than the trapping rate) because of their deformability and structural instability.

The trapping region was reset using an elevated flow rate in the microfluidic channel, allowing the device to be reusable. A temporary increase in the flow rate induced a liposome explosion. Initially, a monodisperse liposome array was produced at flow rates of $(\mathrm{Q} 1, \mathrm{Q} 2)=(1300$ to $330 \mu \mathrm{L} / \mathrm{h}, 300$ to $30 \mu \mathrm{L} / \mathrm{h})$ in $11 \mathrm{~min}$ (Figure S5a). Then, trapped liposomes were completely flushed out within $5 \mathrm{~min}$ at flow rates of $(\mathrm{Q} 1, \mathrm{Q} 2)=(4000 \mu \mathrm{L} / \mathrm{h}$, 0) (Figure S5b). Finally, a liposome array was successfully reproduced by the second loading procedure at flow rates of $(\mathrm{Q} 1, \mathrm{Q} 2)=(1300$ to $330 \mu \mathrm{L} / \mathrm{h}, 300$ to $30 \mu \mathrm{L} / \mathrm{h})$ in $11 \mathrm{~min}$ (Figure S5c).

(2) B. Dura, Y. Liu and J. Voldman, Lab Chip, 2014, 14, 2783-2790. 